\title{
Efficacy of a coaxial system with a compliant balloon catheter for navigation of the Penumbra reperfusion catheter in tortuous arteries: technique and case experience
}

\author{
Kazuki Takahira, MD, ${ }^{1}$ Taketo Kataoka, MD, ${ }^{2}$ Tatsuya Ogino, MD, ${ }^{1}$ Hideki Endo, MD, ${ }^{1}$ and \\ Hirohiko Nakamura, MD, PhD' \\ 1Department of Neurosurgery, Center for Endovascular Neurosurgery, Nakamura Memorial Hospital, Sapporo; and 2Department \\ of Neurosurgery, Kojinkai Social Medical Corporation, Kushiro, Hokkaido, Japan
}

OBJECTIVE The authors describe a method by which they easily and atraumatically navigate a large-bore reperfusion catheter of the Penumbra system to an embolus by using a coaxial system with a compliant balloon catheter in patients with tortuous arteries.

\begin{abstract}
METHODS A retrospective review of the prospective endovascular database was performed to identify cases in which a coaxial system with a compliant balloon catheter (Scepter C, MicroVention/Terumo; or TransForm C, Stryker Neurovascular) and a large-bore reperfusion catheter of the Penumbra system (Penumbra, Inc.) was used. The authors achieved a stable guiding sheath position and delivered the coaxial system with a compliant balloon catheter and a large-bore reperfusion catheter. Then, the balloon was inflated somewhat when the distal tip of the balloon was slightly advanced from the tip of the reperfusion catheter, and together the coaxial system was advanced to an embolus over a 0.014 -in guidewire, even around the corner. When the distal tip of the balloon catheter reached the embolus, the authors deflated the balloon and navigated the large-bore reperfusion catheter to the embolus. Finally, the aspiration of the embolus with the Penumbra MAX pump was begun.
\end{abstract}

RESULTS Between May 2014 and September 2015, the authors used this technique in 17 cases: 16 cases of middle cerebral artery occlusion (including 5 cases of internal carotid artery occlusion) and 1 case of basilar artery occlusion (age range 36-88 years, mean age 74.7 years, $13 \mathrm{men}$ ). For the reperfusion catheter of the Penumbra system, the 5MAX ACE was used in 15 cases, and the 5MAX was used in 2 cases. As a compliant balloon catheter, the Scepter $C$ was used in 16 cases, and the TransForm $C$ was used in 1 case. The technique was successful in 16 cases (94.1\%). No parent artery dissections were noted in any cases. Catheter-induced vasospasm was noted in 1 case, but the vasospasm was transient.

CONCLUSIONS A coaxial system with a compliant balloon catheter can help safely and easily to navigate the largebore reperfusion catheter of the Penumbra system to an embolus in patients with tortuous arteries.

https://thejns.org/doi/abs/10.3171/2016.3.JNS152790

KEY WORDS coaxial system; compliant balloon catheter; reperfusion catheter; Penumbra system; aspiration; vascular disorders; diagnostic and operative techniques

$\mathrm{F}$ OR patients with acute stroke due to major intracranial vessel occlusion, a direct aspiration first pass technique (ADAPT) with a large-bore reperfusion catheter of the Penumbra system (Penumbra, Inc.) is an efficient management strategy and does not increase the incidence of symptomatic intracranial hemorrhage. ${ }^{3}$ However, when we used the 3MAX reperfusion catheter as an inner catheter in cases of severe tortuosity of the vasculature, it could be impossible or hazardous to advance the large-bore reperfusion catheter of the Penumbra system to an embolus along the outer curvature of the vessel in tortuous arteries, mainly because of the gap between the 2 catheters at the distal tip. We describe our experiences in a series of cases in which we used a coaxial system with a

ABBREVIATIONS ADAPT $=$ a direct aspiration first pass technique; $B A=$ basilar artery; ICA $=$ internal carotid artery; $M C A=$ middle cerebral artery; OphA $=$ ophthalmic artery. 
compliant balloon catheter and the large-bore reperfusion catheter of the Penumbra system designed to decrease the gap between the 2 catheters. We have named this strategy the "Rocket" technique, because the edge of this coaxial system appears to have the shape of a rocket.

\section{Methods}

A retrospective review of our prospective endovascular database identified 17 cases treated between May 2014 and September 2015 in which we used the Rocket technique. All patients had tortuosity of the extracranial or intracranial artery. We did not try the standard technique with a large-bore reperfusion catheter and a 3MAX reperfusion catheter in any of the 17 cases.

\section{Operative Technique}

Initial angiography was typically performed through a 4-Fr diagnostic catheter positioned within the proximal parent artery (Fig. 1). This diagnostic catheter was then exchanged, along with the femoral sheath, for a 6-Fr long sheath (KSAW Shuttle; Cook, Inc.) or a 9-Fr balloonguiding catheter (Optimo; Tokai Medical Products, Inc.), which was positioned within the proximal parent artery. Then, we used the coaxial system of a compliant balloon catheter (Scepter C, MicroVention/Terumo; or TransForm C, Stryker Neurovascular) and a $5 \mathrm{MAX}$ ACE or 5MAX reperfusion catheter of the Penumbra system (Penumbra, Inc.) to decrease the gap between the 2 catheters at the distal tip along the outer curvature of the vessel. We achieved a stable guiding sheath position and then delivered the coaxial system with a compliant balloon catheter and reperfusion catheter. Then, the balloon was inflated somewhat when the tip of the balloon was advanced slightly from the tip of the reperfusion catheter, while referring to the markers of the balloon on angiographic studies. We advanced the coaxial system together over a 0.014 -in guidewire even around the corner, while taking care not to penetrate an embolus with the guidewire. When the distal tip of the balloon catheter reached the embolus, we deflated the balloon

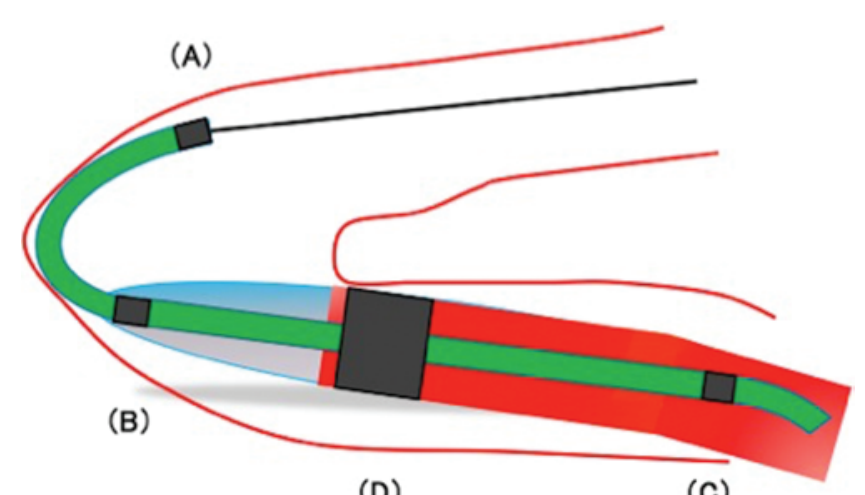

(D)

(C)

FIG. 1. This drawing demonstrates the Rocket technique as performed using angiographic studies. Four markers are detected and are labeled as follows. The distal tip marker of the balloon catheter (A)-only Scepter, exclusive of TransForm; the distal marker of the balloon (B); the proximal marker of the balloon $(C)$; and the tip marker of the reperfusion catheter (D). Figure is available in color online only. and navigated the large-bore reperfusion catheter to the embolus over the balloon catheter and the 0.014-in guidewire. In using Scepter C, we could detect the distal tip of the balloon catheter on angiographic studies. Finally, we began the aspiration of the embolus with the Penumbra MAX pump.

\section{Results}

We used the Rocket technique to perform ADAPT in 17 cases (patient age range $36-88$ years, mean patient age 74.7 years, 13 men): 16 cases with middle cerebral artery (MCA) occlusion and 1 case with basilar artery (BA) occlusion (Table 1). In 5 cases we used this technique to manage MCA occlusion after forced suction by a 9-Fr balloon-guiding catheter for internal carotid artery (ICA) occlusion. For the reperfusion catheter of the Penumbra system, 5MAX ACE was used in 15 cases, and $5 \mathrm{MAX}$ was used in 2 cases. As a compliant balloon catheter, Scepter C was used in 16 cases (Figs. 2 and 3 ) and TransForm $C$ was used in 1 case. All patients had tortuosity of the extracranial or intracranial artery. The technique was successful in 16 cases (94.1\%); in 1 case we could not navigate the coaxial system with Scepter C and 5MAX ACE (Fig. 3). The technique with Scepter $C$ was successful at the first attempt in each case, and the 1 case in which TransForm $\mathrm{C}$ was used was successful on a second attempt. Boluses of calcium channel blockers were not used in any of the cases. Parent artery dissection was not noted in any cases. Catheter-induced vasospasm was noted in 1 case, but this vasospasm was transient.

\section{Discussion}

This case series demonstrated a new technique to easily and atraumatically navigate a large-bore reperfusion catheter to an embolus in patients with tortuous arteries. In patients with acute stroke due to major intracranial vessel occlusion, ADAPT with a large-bore reperfusion catheter of the Penumbra system is an efficient management strategy and does not increase the incidence of symptomatic intracranial hemorrhage. ${ }^{3}$ However, in cases of severe tortuosity of the vasculature, it can be impossible or hazardous to navigate a large-bore reperfusion catheter of the Penumbra system to an embolus when a 3 MAX reperfusion catheter is used as the inner catheter, mainly because of the gap between the 2 catheters at the distal tip. We often had failed to navigate a large-bore reperfusion catheter with a $3 \mathrm{MAX}$ reperfusion catheter in the patients with tortuous anatomy before this study.

When a coaxial system with a $5 \mathrm{MAX}$ reperfusion catheter and a 3MAX reperfusion catheter are used, the gap between the 2 catheters at the distal tip is 0.004 in, because the inner diameter of the 5MAX reperfusion catheter is 0.054 in and the outer diameter of the 3MAX reperfusion catheter is $0.050 \mathrm{in}$. When using the 5MAX ACE reperfusion catheter and the 3MAX reperfusion catheter, the gap is $0.010 \mathrm{in}$. This gap has no impact in a straight vessel, but in the context of a tortuous artery, it can make it difficult and sometimes impossible to navigate a large-bore reperfusion catheter to an embolus, especially around a corner (Fig. 4), because the large-bore reperfusion catheter can 
TABLE 1. Characteristics and procedural details in 17 patients with occlusion of tortuous arteries

\begin{tabular}{|c|c|c|c|c|c|c|c|c|c|c|}
\hline $\begin{array}{l}\text { Case } \\
\text { No. }\end{array}$ & $\begin{array}{l}\text { Age } \\
\text { (yrs) }\end{array}$ & Sex & Location & $\begin{array}{l}\text { NIHSS } \\
\text { Score }\end{array}$ & Catheter & $\begin{array}{c}\text { Reperfusion } \\
\text { Catheter }\end{array}$ & $\begin{array}{l}\text { Balloon } \\
\text { Catheter }\end{array}$ & Guidewire & TICI Score* & Complication \\
\hline 1 & 84 & M & Rt MCA & 14 & Shuttle 6-Fr & $5 M A X$ & Scepter C & Traxcess 0.014 & $2 b$ & None \\
\hline 2 & 78 & M & $\mathrm{BA}$ & 37 & Shuttle 6-Fr & $5 M A X$ & Scepter C & Synchro2 Soft 0.014 & $2 a$ & None \\
\hline 3 & 80 & $M$ & Lt MCA & 26 & Shuttle 6-Fr & 5MAX ACE & Scepter C & Synchro2 Soft 0.014 & 3 & None \\
\hline 4 & 80 & $M$ & Rt MCA & 18 & Shuttle 6-Fr & 5MAX ACE & Scepter C & Synchro2 Soft 0.014 & $2 b$ & None \\
\hline 5 & 82 & M & Lt MCA & 23 & Shuttle 6-Fr & 5MAX ACE & Scepter C & ASAHI CHIKAI 0.014 & 3 & None \\
\hline 6 & 71 & M & Rt MCA & 19 & Shuttle 6-Fr & 5MAX ACE & TransForm C & Transend EX Soft Tip 0.014 & 3 & None \\
\hline 7 & 67 & $\mathrm{~F}$ & Rt MCA & 14 & Shuttle 6-Fr & 5MAX ACE & Scepter C & Synchro2 Soft 0.014 & 3 & None \\
\hline 8 & 36 & $\mathrm{~F}$ & Lt MCA & 9 & Shuttle 6-Fr & 5MAX ACE & Scepter C & Synchro2 Soft 0.014 & 3 & Slight vasospasm \\
\hline 9 & 72 & M & Rt MCA & 3 & Shuttle 6-Fr & 5MAX ACE & Scepter C & Synchro2 Soft 0.014 & 3 & None \\
\hline 10 & 80 & $\mathrm{~F}$ & Lt ICA & 31 & Optimo 9-Fr & 5MAX ACE & Scepter C & Synchro2 Soft 0.014 & $2 b$ & None \\
\hline 11 & 88 & M & Lt ICA & 6 & Optimo 9-Fr & 5MAX ACE & Scepter C & Synchro2 Soft 0.014 & $2 b$ & None \\
\hline 12 & 64 & M & Lt ICA & 17 & Optimo 9-Fr & 5MAX ACE & Scepter C & Synchro2 Soft 0.014 & $2 b$ & None \\
\hline 13 & 86 & $M$ & Lt ICA & 20 & Optimo 9-Fr & 5MAX ACE & Scepter C & ASAHI CHIKAI 0.014 & $2 b$ & None \\
\hline 14 & 65 & M & Lt MCA & 24 & Shuttle 6-Fr & 5MAX ACE & Scepter C & ASAHI CHIKAI 0.014 & 3 & None \\
\hline 15 & 66 & $\mathrm{~F}$ & Lt MCA & 11 & Shuttle 6-Fr & 5MAX ACE & Scepter C & ASAHI CHIKAI 0.014 & 3 & None \\
\hline 16 & 83 & M & Rt MCA & 15 & Shuttle 6-Fr & 5MAX ACE & Scepter C & ASAHI CHIKAI 0.014 & 3 & None \\
\hline 17 & 88 & $M$ & Rt ICA & 22 & Optimo 9-Fr & 5MAX ACE & Scepter C & ASAHI CHIKAI 0.014 & $2 a$ & None \\
\hline
\end{tabular}

$\mathrm{NIHSS}=$ National Institutes of Health Stroke Scale; $\mathrm{TICl}=$ thrombolysis in cerebral infarction.

* According to Higashida et al.
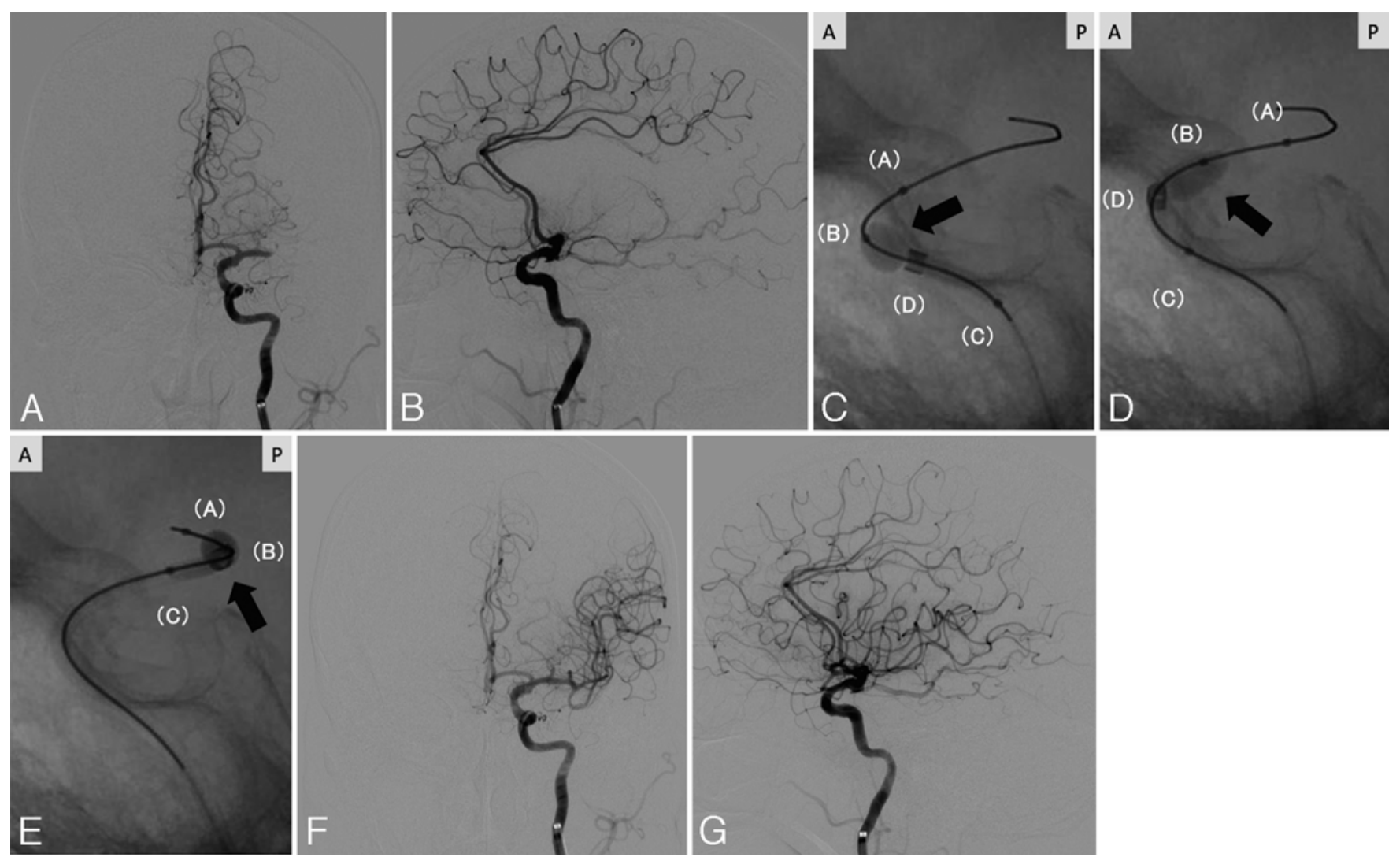

FIG. 2. Case 5. Angiography obtained in an 82-year-old man with left MCA occlusion. Left ICA angiogram demonstrated moderate tortuosity (A and B). A Scepter C $4 \times 10-\mathrm{mm}$ balloon (arrows) was inflated in the horizontal segment of the ICA, and a coaxial system with a 5MAX ACE and a Scepter $\mathrm{C}$ was easily navigated over an ASAHI CHIKAI (Asahi Intecc Co., Ltd.) 0.014-in guidewire (C-E). Following treatment, angiography demonstrated no evidence of carotid dissection or injury (F and $\mathbf{G})$. See Fig. 1 legend for definitions of the markers in parentheses in panels $\mathrm{C}-\mathrm{E}$. 

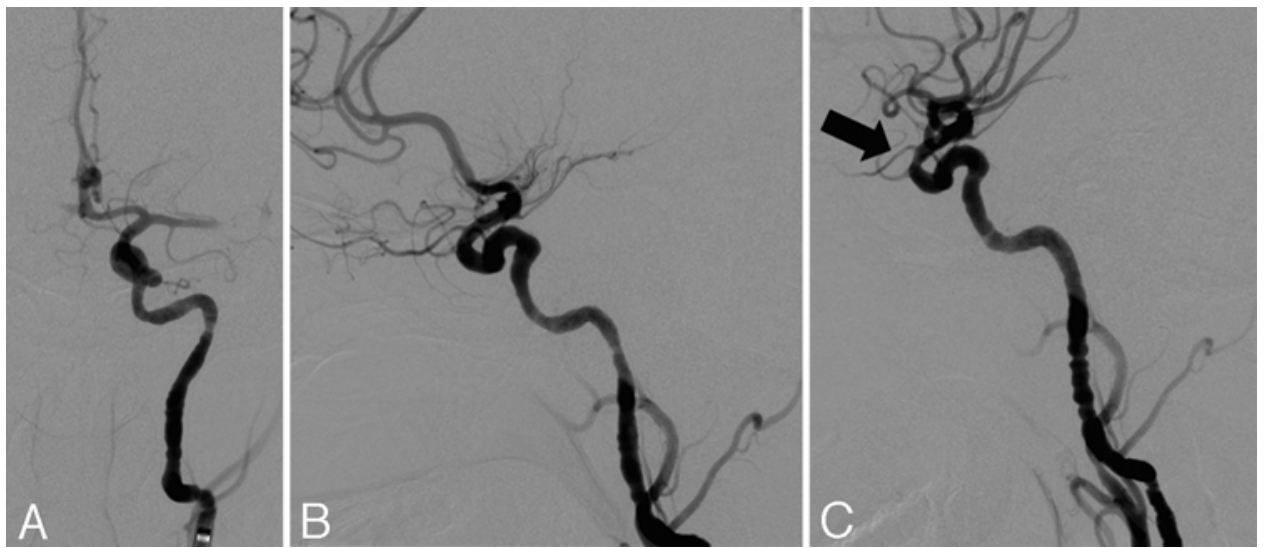

FIG. 3. Case 8. Angiography obtained in a 36-year-old woman with left MCA occlusion. Left ICA angiogram demonstrated severe tortuosity $(A$ and $B$ ). We tried to navigate a coaxial system with a Scepter $C 4 \times 10-\mathrm{mm}$ balloon and 5MAX ACE, but were unsuccessful due to significant stress on the vessel wall at the corner near the ophthalmic artery (OphA). After using steam to shape the tip of the 5MAX ACE along the curvature, we could navigate the coaxial system over a Synchro2 soft 0.014-in guidewire (Stryker Neurovascular) to the embolus. Following treatment, angiography demonstrated mild vasospasm (arrow) at the distal part of the OphA, but no evidence of carotid dissection (C). The vasospasm improved immediately.

be advanced along the outside of the inner catheter. Even if it is possible to advance the inner catheter toward the periphery, it can be hazardous or impossible to advance the large-bore reperfusion catheter because the stress due to the gap damages the vessel wall, and traumatic injury happens as a result of this damage. This problem can increase the technical difficulty, the procedure time, and the risk of complications. Therefore, we did not try the standard technique in all 17 cases at first.

A coaxial system with a compliant balloon catheter can address these technical limitations. First, the placement of a compliant balloon that fills the gap between the 2 catheters at the distal tip can reduce the stress along the vessel wall and enable the safe advancement of a largebore reperfusion catheter around the corner. A compliant balloon is so soft and supple that it can reduce the risk of vasospasm and traumatic injury, even in severely tortuous vessels. The degree of balloon inflation can also be modi- fied to avoid damaging a large-bore catheter and the vessel wall. Also, the presence of a distal tip in a compliant balloon catheter is important. The length of the distal tip is 5 $\mathrm{mm}$ in Scepter C and $3.25 \mathrm{~mm}$ in TransForm C. By using the length, the coaxial system can be navigated together even around the corner, as if it were a single catheter. Also, in using Scepter C, the distal tip marker of the balloon catheter can help visualization on angiographic studies, to avoid penetrating an embolus. Finally, the balloon catheter can be navigated beyond the tortuous anatomy over a 0.014-in guidewire. A 0.014-in guidewire might be better than a 0.010 -in guidewire in advancing the coaxial system together, because the 0.014-in guidewire has superior operability and trackability. When the balloon is deflated, the large-bore reperfusion catheter can be gently guided to the embolus over the balloon catheter and the 0.014-in guidewire.

A similar concept has not been described for acute
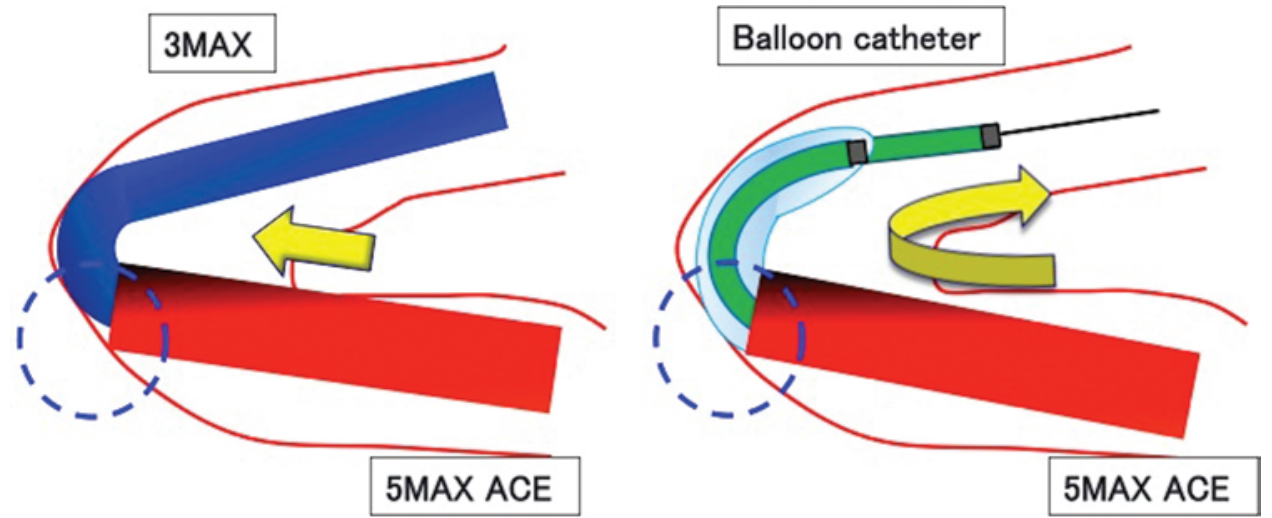

FIG. 4. These drawings demonstrate the structures of the coaxial system in each pattern at the curvature of the vessel. Left: When using the 3MAX reperfusion catheter, the gap between the 2 catheters often creates stress on the vessel wall (straight arrow), and it is hazardous or impossible to navigate a large-bore reperfusion catheter to an embolus. Right: When using the Scepter $\mathrm{C}$, filling the balloon can reduce the gap between the 2 catheters, and it is possible to navigate a large-bore reperfusion catheter easily even at the curvature of a tortuous artery (curved arrow). The dashed circles designate the vessel wall area under stress. Figure is available in color online only. 
stroke treatment, but a novel technique has been reported for navigation of a catheter with a compliant balloon catheter in cases of a tortuous artery. Peeling and Fiorella ${ }^{2}$ used balloon-assisted guide catheter positioning to overcome extreme cervical carotid tortuosity. Those investigators used a compliant balloon (Scepter, HyperForm, HyperGlide; ev3, Covidien) as an anchor to achieve distal positioning of a flexible guiding catheter beyond the extreme cervical carotid tortuosity when performing coil embolization for an intracranial aneurysm in the anterior circulation. When using this technique, there were no perioperative complications (e.g., vasospasm or vascular damage) in any of the 12 patients, and hypercompliant balloon catheters could be reliably used to facilitate safe and rapid distal positioning of the flexible guiding catheters beyond the severe cervical carotid tortuosity. In our cases, parent artery dissections were not noted, and catheter-induced vasospasm was mild and transient in 1 case. These data suggest that the Rocket technique, in which a compliant balloon catheter is used, can be an effective and safe strategy for the management of acute stroke.

The Rocket technique has several limitations. First, a large-bore reperfusion catheter of the Penumbra system can be damaged at the distal tip or at other parts. In 1 case, slight damage was noted at the distal tip of the reperfusion catheter, which might have resulted from overexpansion of the balloon. The damage to other portions of the reperfusion catheter might be due to the gap between the 2 catheters or to the catheters' stiffness. Second, the compliant balloon can be damaged due to the stress on the vessel wall in advancing the coaxial system. Third, this procedure can require significant technical skill on the part of the operators. Finally, this technique may be associated with a slightly longer procedural time and an increased risk of traumatic injury or vasospasm, because increasing the stability of the whole construction by inflating a balloon may make navigation more difficult.

In 1 case (Case 8), we could not navigate the coaxial system with the Scepter C and the 5MAX ACE reperfusion catheter (Fig. 3). The patient was a 36-year-old woman who had severe tortuosity of the ICA. In most previous cases similar to this one, we put out the balloon as much as one-third of its length from the tip of the reperfusion catheter and navigated the coaxial system together after inflating the balloon slightly to reduce the gap between the 2 catheters. But the balloon was bent at the corner of the vessel, and advancing the coaxial system could create significant stress on the vessel wall. In this case, the stress against the vessel wall precluded navigation of the coaxial system at the distal part of the ophthalmic artery (OphA), and mild catheter-induced vasospasm occurred at the corner. After using steam to shape the tip of the 5MAX ACE along the curvature, we could navigate the coaxial system together to the embolus. Thereby, we recognized that it was important when we inflated a balloon that we put it out only slightly, or less than one-third of its length from the tip of the reperfusion catheter. When doing so, the Rocket technique was successful in all subsequent cases after Case 8, and catheter-induced vasospasm did not occur in any of the remaining 9 cases. Additional cases of intracranial major vessel occlusion with tortuous arteries are needed to further refine the Rocket technique, because we studied only 17 cases in this series.

\section{Conclusions}

A coaxial system with a compliant balloon catheter was effective and safe for navigation of a large-bore reperfusion catheter of the Penumbra system to the embolus in patients with tortuous arteries.

\section{Acknowledgments}

We express the deepest appreciation to individuals who contributed to the study or to manuscript preparation.

\section{References}

1. Higashida RT, Furlan AJ, Roberts H, Tomsick T, Connors B, Barr J, et al: Trial design and reporting standards for intraarterial cerebral thrombolysis for acute ischemic stroke. Stroke 34:e109-e137, 2003

2. Peeling L, Fiorella D: Balloon-assisted guide catheter positioning to overcome extreme cervical carotid tortuosity: technique and case experience. J Neurointerv Surg 6:129133,2014

3. Turk AS, Frei D, Fiorella D, Mocco J, Baxter B, Siddiqui A, et al: ADAPT FAST study: a direct aspiration first pass technique for acute stroke thrombectomy. J Neurointerv Surg 6:260-264, 2014

\section{Disclosures}

The authors report no conflict of interest concerning the materials or methods used in this study or the findings specified in this paper.

\section{Author Contributions}

Conception and design: Takahira, Kataoka. Acquisition of data: Takahira. Analysis and interpretation of data: Takahira. Drafting the article: Takahira. Administrative/technical/material support: Kataoka, Endo, Ogino. Study supervision: Kataoka, Nakamura.

\section{Correspondence}

Kazuki Takahira, Nakamura Memorial Hospital, Department of Neurosurgery, South 1, West 14, Chuo-ku, Sapporo 0608570, Japan.email: tkhrkzk@gmail.com. 